\title{
Radiomics Signature: A potential biomarker for the prediction of survival in Advanced Hepatocellular Carcinoma
}

\author{
Lingli $\mathrm{Li}^{1,2^{*}}$, Xuefeng Kan ${ }^{1,2^{*}}$, Yongjun Zhao ${ }^{3}$, Bo Liang ${ }^{1,2}$, Tianhe Ye ${ }^{1,2}$, Lian Yang ${ }^{1,2 \bowtie}$ and Chuansheng Zheng ${ }^{1,2 \bowtie}$ \\ 1. Department of Radiology, Union Hospital, Tongji Medical College, Huazhong University of Science and Technology, Wuhan 430022, China. \\ 2. Hubei Key Laboratory of Molecular Imaging, Union Hospital, Tongii Medical College, Huazhong University of Science and Technology, Wuhan 430022 , China. \\ 3. Wuhan Zhikai Technology, Wuhan 430074, China. \\ *These authors contributed equally to this work. \\ $\triangle$ Corresponding authors: Lian Yang, Department of Radiology, Union Hospital, Tongji Medical College, Huazhong University of Science and Technology, 1277 Jiefang \\ Avenue, Wuhan 430022, China. Tel.: +86-027-84289536; Fax: 086-27-85727001; E-mail: yanglian@hust.edu.cn; Chuansheng Zheng, Department of Radiology, Union Hospital, \\ Tongji Medical College, Huazhong University of Science and Technology, 1277 Jiefang Avenue, Wuhan 430022, China. Tel: +86-027-84289536; Fax: 086-27-85727001; E-mail: \\ hqzcsxh@sina.com.
}

(C) The author(s). This is an open access article distributed under the terms of the Creative Commons Attribution License (https://creativecommons.org/licenses/by/4.0/). See http://ivyspring.com/terms for full terms and conditions.

Received: 2020.11.05; Accepted: 2021.03.15; Published: 2021.03.30

\begin{abstract}
Objectives: To develop and validate radiomics nomograms for the pretreatment predictions of overall survival (OS) and time to progression (TTP) in the patients with advanced hepatocellular carcinoma (HCC) treated with apatinib plus transarterial chemoembolization (TACE), and to assess the incremental value of the clinical-radiomics nomograms for estimating individual OS and TTP.

Methods: A total of 60 patients with advanced HCC (BCLC stage C) treated with apatinib plus TACE were divided into a training set $(n=48)$ and a validation set $(n=12)$. The predictors identified from the clinical variables and the radiomics signature constructed from the computed tomography images, such as a-fetoprotein level (AFP), formfactor, the grey level co-occurrence matrix, the gray level size zone matrix, and the gray level run-length matrix, were used to build the clinical-radiomics nomograms and the radiomics nomograms for the prediction of OS and TTP.

Results: Apatinib plus TACE benefited the patients with advanced HCC, with a 579-day median OS and a 270-day median TTP. The nomograms were built with the radiomics signature and AFP, and achieved favorable prediction efficacy with acceptable calibration curves. Decision curve analyses demonstrated that the clinical-radiomics nomograms outperformed the radiomics nomograms for the predictions of OS and TTP.

Conclusions: Apatinib plus TACE may improve OS and prolonged TTP in the patients with advanced HCC. The clinical-radiomics nomograms, a noninvasive pretreatment prediction tool that incorporate radiomics signature and AFP, demonstrated good prediction accuracy for OS and TTP in these patients. These results indicate that the clinical-radiomics nomograms may provide novel insight for precise personalized medicine approaches in the patients with advanced HCC.
\end{abstract}

Key words: radiomics, nomogram, hepatocellular carcinoma, survival

\section{Introduction}

Hepatocellular carcinoma (HCC) is a complex disease most commonly related to chronic liver disease. Epidemiological studies of HCC have revealed that it is the fifth most common cancer and the second most frequent cause of cancer-related death [1], with a growing incidence worldwide [2]. HCC also represents about $90 \%$ of primary liver cancers [2], with a male to female ratio of approximately 2-2.5:1 [3]. The greatest HCC burden is in the developing world [4]. Approximately $25-70 \%$ of patients with HCC present with advanced disease at diagnosis, which is regarded as incurable [2, 4]. Treatment selection and survival prediction are critical steps in the management of advanced HCC (BCLC stage C).

Targeted drug therapy is the most common treatment for advanced HCC. Sorafenib, which inhibits vascular endothelial growth factor receptors, 
is regarded as the standard first-line systemic therapy for advanced HCC [2]. However, the incidence of drug-related adverse events is high, and these adverse events may reduce adherence to sorafenib and thus have a negative effect on patient prognosis [5].

In addition to its association with adverse events, the high cost of sorafenib also limits its long-term use in the treatment of advanced HCC patients. For these reasons, new targeted drugs for advanced HCC are currently being developed and tested. For instance, apatinib is a novel and highly selective inhibitor of VEGFR2 tyrosine kinase, with a binding affinity 10 times greater than that of sorafenib [6]. Apatinib is currently available in mainland China. A series of studies [7-9] found that apatinib has encouraging antitumor properties and is toxicologically tolerable in several malignant tumor cases. Furthermore, another study [10] found that apatinib has similar antitumorigenic and antiangiogenic efficacy to sorafenib in HCC with less toxicity in vitro and in vivo. These findings provide preclinical evidence supporting the potential application of apatinib to the treatment of HCC. What's more, apatinib has potential survival benefits for patients with advance HCC, as demonstrated by a phase II randomized, open-label trial [11].

In recent years, the combined use of transarterial chemoembolization (TACE) with anti-angiogenic agents in advanced HCC patients has attracted much interest [12-16]. Our previous study [17] also showed the median time to progression (TTP) and overall survival (OS) in the TACE-apatinib group was also significantly greater than that of the TACE-alone group after the propensity score matching analysis, which agreed with those of a series of previous studies [12-14]. Apatinib plus TACE had certain survival benefits for advanced HCC in patients who experienced progression following TACE, revealing a potentially promising strategy for the treatment of advanced HCC [12]. Given this, determining biomarkers predictive of the efficacy of apatinib plus TACE is needed.

Radiomics is an emerging field in which highdimensional features are mathematically extracted from medical images. This extraction results in the conversion of images into mineable data and the subsequent analysis of these data for support of medical decision-making [18]. Such prognostic prediction models may be built from noninvasively extracted radiomics features of tumors and relevant clinical indicators. Recently, radiomics analyses of HCC using computed tomography (CT) and magnetic resonance images have been shown to have high prediction accuracy [19-21], such as Kim et al. [20] found that gadoxetic acid-enhanced magnetic resonance imaging radiomics could be used for the prediction of postoperative early and late recurrence of single HCC. These radiomic analysis of contrast-enhanced CT predictors have mainly based on advanced HCC patients who were treated with sorafenib [21] or microvascular invasion and outcome in hepatocellular carcinoma [19]. However, the use of texture analysis as a therapeutic decision-making biomarker or predictive biomarker for treatment efficacy in cases of advanced HCC treated with apatinib plus TACE has never been investigated.

The aim of present study was to develop and validate radiomics nomograms for the pretreatment predictions of OS and TTP in patients with advanced HCC who were treated with apatinib plus TACE, and to assess the incremental value of such clinical-radiomics nomograms in individual OS and TTP estimation.

\section{Materials and methods}

\section{Subjects}

Ethical approval by the institutional review board was obtained for this retrospective analysis and the requirement for informed consent was waived. This study was conducted in accordance with the Declaration of Helsinki.

Between January 2014 and June 2018, a total of 60 consecutive patients with advanced HCC (BCLC stage C) who received apatinib plus TACE were enrolled in the present study. Patient recruitment (Figure S1) and inclusion and exclusion criteria are depicted in the Supplementary Data. A detailed version of the apatinib plus TACE administration protocol used here is also given in the Supplementary Data. Computer-generated random numbers were used to assign a training set to validation set ratio of about 4:1. Recorded demographic characteristics and clinical data are detailed in the Supplementary Data.

\section{End Points}

End points were chosen according to the guidelines of the American Association for the Study of Liver Diseases [22]. The primary end point was OS, defined as the time from the first TACE procedure to the patient's death. TTP (the time from the start of the first TACE procedure to the time of tumor progression as defined by modified Response Evaluation Criteria in Solid Tumors [23]) was defined as the secondary end point. Patient follow-up is detailed in the Supplementary Data. Patients alive or those without radiologic progression at the end of the follow-up period were removed. 


\section{Median OS and median TTP}

Median OS and median TTP of the full cohort (all 60 patients) were calculated using Kaplan-Meier analyses.

\section{CT image acquisition, region-of-interest segmentation, and radiomics features extraction}

The radiomics workflow used in the present study is depicted in Figure 1. A detailed CT protocol is also given in the Supplementary Data. Tumor regions of interest (ROIs) were hand-drawn on both the late arterial phase and the portal venous phase of pretreatment contrast-enhanced CT images on each slice by two radiologists with more than 10 years of experience. Itk-SNAP software (www.itk-snap.org) was used for manual segmentation. Radiomics features for each patient were extracted using Artificial Intelligence Kit version 1.0.3 (GE Healthcare, Boston USA). In total, 396 texture parameters from the late arterial phase and 396 texture parameters from the portal venous phase were extracted from a single CT image of each patient. The radiomics features were classified into six categories (Supplementary Table
S1): histogram, the grey level co-occurrence matrix (GLCM), the gray level size zone matrix (GLSZM), the gray level run-length matrix (RLM), and shape- and size-based features. More detailed information about the radiomics features can be found in the Supplementary Data.

Intra- and inter-reader agreement for the texture parameters were assessed using intraclass correlation coefficients (ICCs) for each pair of variables in the whole sample. To assess intra-observer reproducibility, reader 1 then repeated the same manual procedure one week later. An ICC greater than 0.7 was considered good feature extraction agreement [21, 24]. Correlations between texture parameters were assessed using Spearman correlations, with correlation coefficients more than 0.9 considered significant.

\section{Feature selection and radiomics signature construction}

The least absolute shrinkage and selection operator (LASSO) Cox regression algorithm was used to analyze all high-dimensional data. A LASSO Cox regression was applied to select OS- and TTP-related
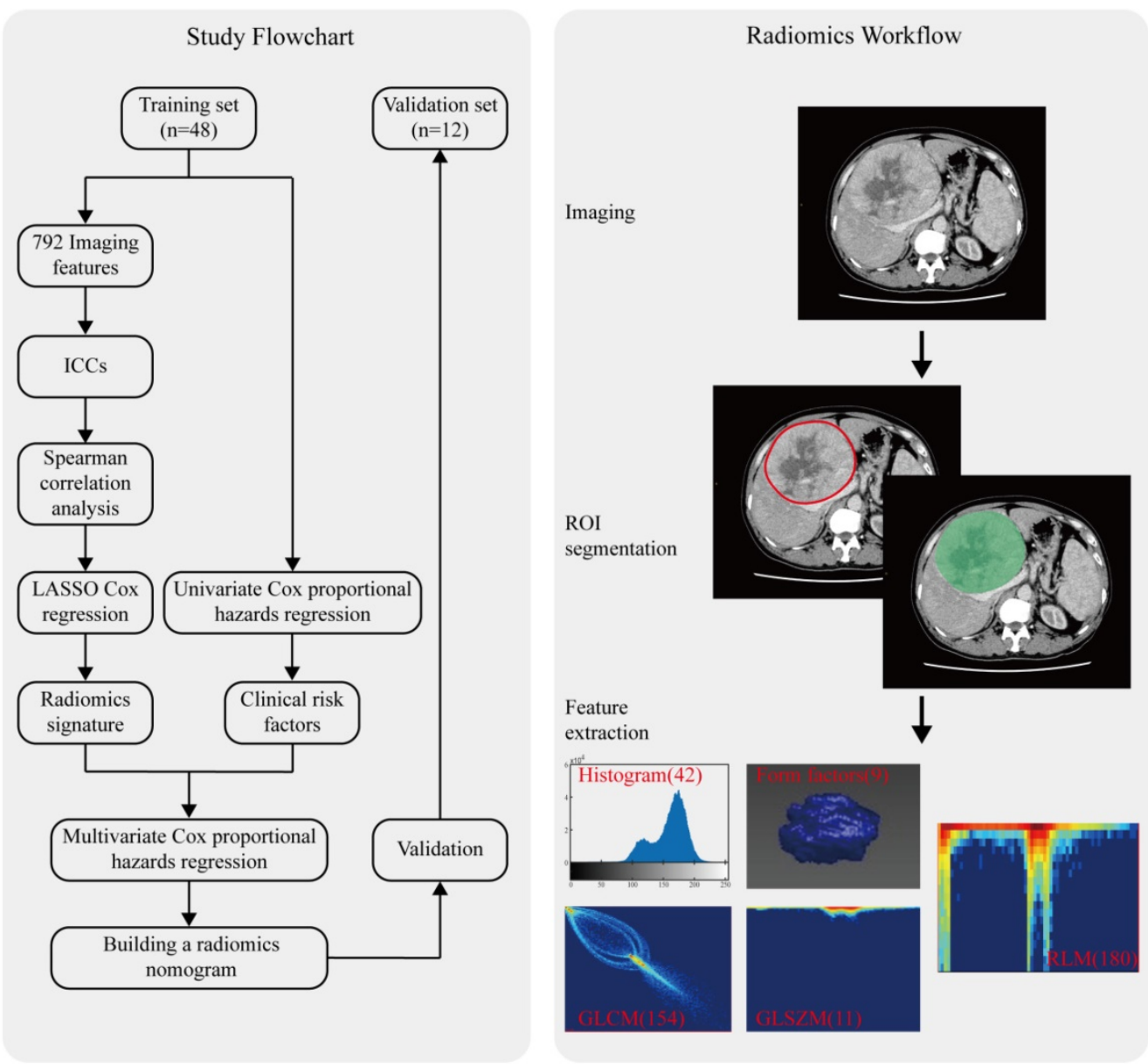

Figure 1. Radiomics workflow and study flowchart. 
features with nonzero coefficients from a subset of features out of the 792 texture parameters in the training set after performing the spearman correlation analysis. Penalty parameter tuning was conducted with 10-fold cross-validation. Additional details on feature processing and selection can be found in our prior work [25]. A radiomics score (Rad-score) was generated using a linear combination of selected features that were weighted by their respective LASSO Cox regression coefficients. The potential survival predictors among clinical variables, such as AFP, total bilirubin, and Child-Pugh class, were identified using a univariate Cox proportional hazards regression analysis approach.

\section{Construction of the radiomics nomogram}

The radiomics signature and clinical predictors were tested via a multivariate Cox proportional hazards regression model to predict OS and TTP in the training set. To provide the clinician with a quantitative tool to predict individual probability of OS and TTP, we built the clinical-radiomics nomograms and radiomics nomograms on the basis of multivariate Cox proportional hazards regression analysis with the training set.

\section{Assessment of nomogram performance}

Nomogram calibration was assessed with a calibration curve. Harrell's C-index was calculated, which was applied to quantify the discrimination performance of the radiomics nomogram. The clinical-radiomics nomograms and radiomics nomograms were subjected to bootstrapping validation $(1,000$ bootstrap resamples) to calculate a relatively corrected C-index [26].

\section{Internal validation of the radiomics nomogram}

The performance of the internally-validated radiomics nomogram was assessed with the validation set. Using the formula constructed in the training set, a Rad-score was calculated for each patient in the validation set. Harrell's C-index and the relatively corrected C-index were calculated, and the resultant calibration curves were obtained.

\section{Clinical utility of the radiomics nomogram}

Decision curve analysis (DCA) was conducted to determine the clinical usefulness of the clinicalradiomics nomograms by calculating the net benefits for a range of threshold probabilities on the full cohort with 5-fold cross-validation [27].

\section{Statistical analyses}

Statistical analyses were conducted with $\mathrm{R}$ software (version 3.5.3, http://www.Rproject.org) and Python3.7. Package details are available in the
Data Supplement. A two-sided $\mathrm{P}<0.05$ was considered significant.

\section{Results}

\section{Patient characteristics and univariate analyses of training set outcomes}

The study flowchart is presented in Figure 1. The main clinical characteristics of patients in the training and validation sets are shown in Table 1 . The median OS in the whole cohort was 579 days (range, 90-1975) and the median TTP was 270 days (range, 30-1006). Demographic and pretreatment clinical characteristics did not significantly differ between the training and validation sets, except for a significantly lower pretreatment serum albumin level and tumor size in the validation set $(\mathrm{P}=0.017, \mathrm{P}=0.018)$. The median $\mathrm{OS}$ was 480 days (range, 90-1975) in the training set and 570 days (range, 180-1468) in the validation set $(\mathrm{P}=$ 0.496). The median TTP in the training and validation sets were 240 (range, 30-1006) and 275 (range, 60-458) days, respectively $(\mathrm{P}=0.28)$.

Table 1. Main baseline demographic and clinical characteristics of patients in the training set and validation set

\begin{tabular}{|c|c|c|c|}
\hline Characteristic & $\begin{array}{l}\text { Training set } \\
(\mathrm{n}=48)\end{array}$ & $\begin{array}{l}\text { Validation set } \\
(\mathrm{n}=12)\end{array}$ & $P$-value \\
\hline Sex & & & 0.998 \\
\hline Male & $41(85)$ & $10(83)$ & \\
\hline Female & $7(15)$ & $2(17)$ & \\
\hline Median age $(y)^{*}$ & $49(31-66)$ & $49(36-59)$ & 0.318 \\
\hline Median BMI $\left(\mathrm{kg} / \mathrm{m}^{2}\right)^{*}$ & $21.9(18.3-28.7)$ & $23.5(18.4-27.9)$ & 0.134 \\
\hline Cause of disease & & & 0.997 \\
\hline Chronic hepatitis B only & $43(90)$ & $11(92)$ & \\
\hline Unknown & $5(10)$ & $1(8)$ & \\
\hline \multicolumn{4}{|l|}{ Child-Pugh class } \\
\hline A & $41(85)$ & $9(75)$ & 0.861 \\
\hline B & $7(15)$ & $3(25)$ & \\
\hline ECOG performance status & & & 0.862 \\
\hline 0 & $7(15)$ & $1(8)$ & \\
\hline 1 & $36(75)$ & $8(67)$ & \\
\hline 2 & $5(10)$ & $3(25)$ & \\
\hline HCC type & & & 0.717 \\
\hline Nodular & $23(48)$ & $8(67)$ & \\
\hline massive & $25(52)$ & $4(33)$ & \\
\hline Median tumor size $(\mathrm{mm})$ & $79(32-160)$ & $60(35-121)$ & $0.018^{\#}$ \\
\hline Macroscopic vascular invasion & $28(58)$ & $6(50)$ & 0.965 \\
\hline Extrahepatic spread & $30(63)$ & $7(58)$ & 0.995 \\
\hline \multicolumn{4}{|l|}{ Biochemical analysis* } \\
\hline Median albumin level (g/dL) & $37(25-45)$ & 34 (31-39) & $0.017 \#$ \\
\hline Median total bilirubin level (mg/dL) & $16(6-41)$ & $19(10-101)$ & 0.216 \\
\hline Median a-fetoprotein level (ng/mL) & $760(1-84500)$ & $336(3-72814)$ & 0.419 \\
\hline \multicolumn{4}{|l|}{ Median survival end points $(\mathrm{d})^{*}$} \\
\hline Overall survival & $480(90-1975)$ & $570(180-1468)$ & 0.496 \\
\hline Time to progression & $240(30-1006)$ & $275(60-458)$ & 0.280 \\
\hline
\end{tabular}

Supplementary Table S2 contains the results of a univariate cox proportional hazards regression 
analyses of pretreatment clinical characteristics, for predicting OS and TTP in the training set. Among the pretreatment demographic and clinical parameters, a-fetoprotein (AFP) level (hazard ratio [HR], 1.000021; 95\% confidence interval [CI]: 1.000007, 1.000035; $\mathrm{P}<0.005)$ was significantly associated with OS. AFP level (HR, 1.00002; 95\% CI: 1.00001, 1.00003; P<0.005) was also significantly associated with TTP.

\section{Feature selection and radiomics signature construction}

The inter- (ICC, range 0.70-0.98) and intraobserver (ICC, 0.70-0.96) ratings were high, indicating favorable intra- and inter-observer feature extraction reproducibility. Given this, all outcomes were based on the measurements obtained by the first radiologist. All features with spearman correlation coefficients greater than 0.9 were removed. A total of 57 radiomics features for each patient were reserved from the late arterial (29 features) and portal venous phases (28 features).

The 57 radiomics features of advanced HCC from the late arterial and venous phases of pretreatment contrast-enhanced CT images were reduced to 8 potential predictors of OS on the basis of 48 patients in the training set (Supplementary Table S3A). These features are of nonzero coefficients in the LASSO logistic regression algorithm. Similarly, 12 potential predictors of TTP were reserved (Supplementary Table S3B). These radiomics features were included in the Rad-score calculation formula (Supplementary Data).

\section{Construction of the radiomics nomogram}

A multivariate Cox proportional hazards regression analysis identified the radiomics signature and AFP as independent predictors (Table 2, Table 3, Supplementary Table S4A-Table S4B). The models that incorporated these independent predictors were developed and presented as the nomograms (Figure 2A-B, Figure 3A-B).

Table 2. Multivariate Cox proportional hazards regression analyses of the advanced HCC radiomics signature and clinical features for predicting overall survival in the training set

\begin{tabular}{lll}
\hline Radiomics signature and clinical feature & Hazard Ratio* & $P$-value \\
\hline $\begin{array}{l}\text { a-fetoprotein level } \\
\text { InverseDifferenceMoment_AllDirection_offset4_SD }\end{array}$ & $0.72(0.47-1.09)$ & 0.12 \\
$\begin{array}{l}\text { (art) } \\
\text { Compactness1 (art) }\end{array}$ & $0.65(0.43-0.97)$ & $0.04^{\#}$ \\
ClusterShade_AllDirection_offset1 (por) & $2.53(1.58-4.48)$ & $<0.005^{\#}$ \\
ZonePercentage (por) & $1.73(1.20-2.48)$ & $<0.005^{\#}$ \\
\hline $\begin{array}{l}\text { Note.-HCC: hepatocellular carcinoma, art: late arterial phase, por: portal venous } \\
\text { phase; }\end{array}$ & & \\
*Numbers in parentheses are 95\% confidence intervals; & & \\
\#Statistically significant. &
\end{tabular}

Table 3. Multivariate Cox proportional hazards regression analyses of the advanced HCC radiomics signature and clinical features for predicting time to progression in the training set

\begin{tabular}{llc}
\hline Radiomics signature and clinical feature & Hazard Ratio* & $P$-value \\
\hline a-fetoprotein level & $1.54(1.05-2.27)$ & $0.03^{\#}$ \\
ShortRunEmphasis_AllDirection_offset1_SD (art) $)$ & $2.33(1.20-4.53)$ & $0.01^{\#}$ \\
Compactness1 (art) & $0.66(0.46-0.93)$ & $0.02^{\#}$ \\
$\begin{array}{l}\text { ShortRunHighGreyLevelEmphasis_AllDirection_ } \\
\text { offset1_SD (art) }\end{array}$ & $1.53(0.30-0.92)$ & $0.03^{\#}$ \\
$\begin{array}{l}\text { LargeAreaEmphasis (art) } \\
\text { LowGreyLevelRunEmphasis_AllDirection_ }\end{array}$ & $0.73(0.48-1.11)$ & 0.13 \\
offset4_SD (art) & & $0.01^{\#}$ \\
\hline $\begin{array}{l}\text { Note.-HCC: hepatocellular carcinoma, art: late arterial phase, por: portal venous } \\
\text { phase; }\end{array}$ & \\
*Numbers in parentheses are 95\% confidence intervals; & \\
\#Statistically significant. & &
\end{tabular}

\section{Apparent performance of the radiomics nomogram in the training set and validation in the validation set}

The calibration curves of the radiomics nomograms and the clinical-radiomics nomograms for OS and TTP demonstrated good agreement between prediction and observation in the training set (Figure 2C-D, Figure 3C-D). The C-index for the clinical-radiomics nomogram-based prediction of OS and TTP for the training set were $0.833(95 \% \mathrm{CI}, 0.793$ to 0.873 ) and 0.739 (95\% CI, 0.692 to 0.786$)$, then confirmed to be $0.792(95 \% \mathrm{CI}, 0.715$ to 0.870$)$ and 0.701 (95\% CI, 0.635 to 0.767$)$ via bootstrapping validation in the validation set, respectively. While the C-index for the radiomics nomogram-based prediction of OS and TTP for the training set were 0.828 (95\% CI, 0.787 to 0.869$)$ and 0.732 (95\% CI, 0.685 to 0.779 ), then confirmed to be 0.745 ( $95 \% \mathrm{CI}, 0.695$ to $0.795)$ and 0.586 (95\% CI, 0.543 to 0.628$)$ via bootstrapping validation in the validation set, respectively.

\section{Clinical utility of the radiomics nomogram}

A decision curve analysis revealed that the clinical-radiomics nomograms developed here had higher overall net benefit than the radiomics nomograms across most of the range of reasonable threshold probabilities for both OS and TPP (Figure $4)$.

\section{Discussion}

This study presents a retrospective analysis to develop and validate radiomics nomograms for the pretreatment predictions of OS and TTP in patients with advanced HCC who were treated with apatinib plus TACE, and to assess the incremental value of such clinical-radiomics nomograms in individual OS and TTP estimation, which is an important complement to these previous studies [19-21]. Collectively, the results of present study suggest that 
apatinib plus TACE may improve OS and prolong TTP in patients with advanced HCC. These results agree with those of a series of previous studies [12-14, 17]. When compared to a median survival time of $6-8$ months or $25 \%$ at one year in patients with advanced HCC (BCLC stage C), as described by the EASL clinical practice guidelines [2], the results of the present study indicate that apatinib plus TACE may improve OS in patients with advanced HCC. Similarly, when compared to a median TTP of 2.8 months in sorafenib-treated patients [28], the results of the present study reveal that apatinib plus TACE may prolong TTP in patients with advanced HCC. Though the present study found that apatinib plus TACE may be a promising strategy for the treatment of advanced HCC, the present study's sample size was relatively small. Future investigations should utilize a larger sample size and multicenter validation

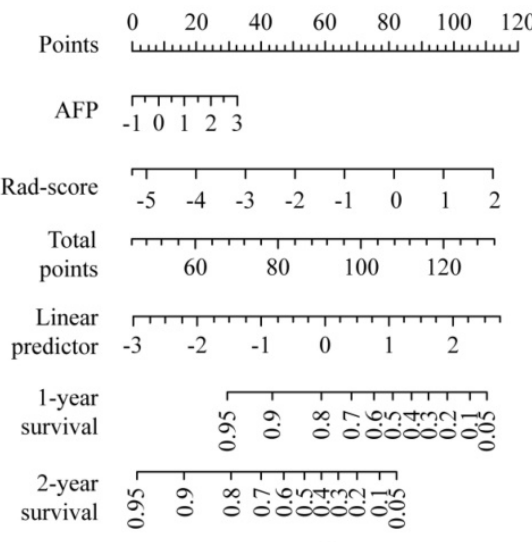

A

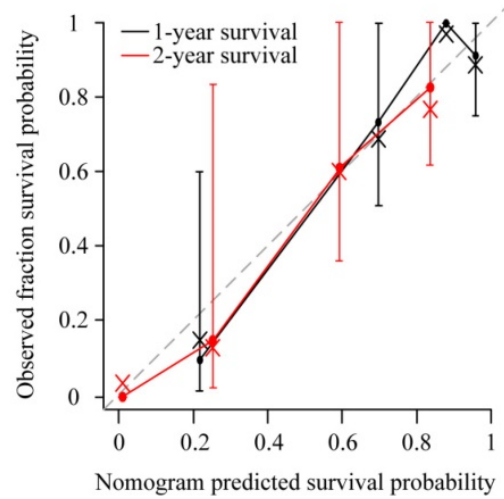

$\mathrm{C}$
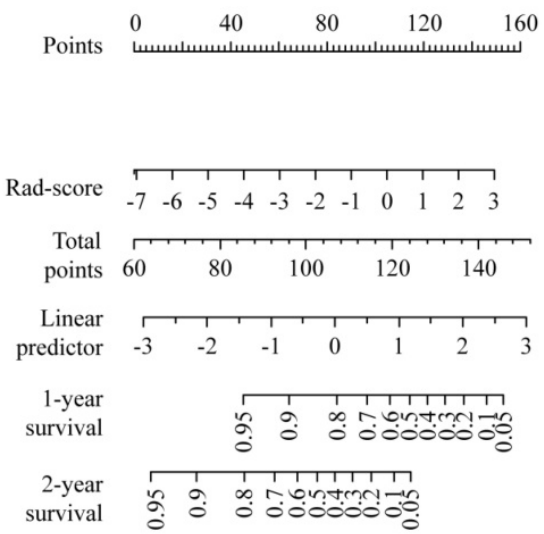

B

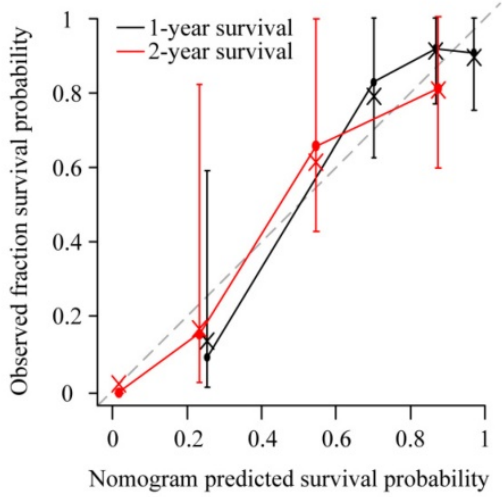

D
Figure 2. Use of the constructed clinical-radiomics nomogram and radiomics nomogram to predict overall survival (OS) in patients with advanced HCC, along with the assessment of the model calibration. Clinical-radiomics nomogram (A) and radiomics nomogram (B). Locate the patient's Rad-score on the Rad-score axis. Draw a line straight upward to the points' axis to determine how many points toward the probability of OS the patient receives for his or her Rad-score. Repeat the process for each variable. Sum the points achieved for each of the risk factors. Locate the final sum on the Total Point axis. Draw a line straight down to find the patient's probability of OS. Calibration curves for the clinical-radiomics nomogram (C) and radiomics nomogram (D) show the calibration of each model in terms of the agreement between the predicted and the observed 1-and 2-year outcomes. Nomogram predicted OS is plotted on the x-axis; the observed fraction OS is plotted on the $y$-axis. Diagonal dotted line $=$ a perfect prediction by an ideal model, in which the predicted outcome perfectly corresponds to the actual outcome. Solid line = performance of the nomogram, a closer lining of which with the diagonal dotted line represents a better prediction. approaches to establish stronger evidence for the identified in present study to be an independent marker for OS and TTP in patients with advanced the radiomics nomograms alone. Prior studies [21, 29, have also shown that several clinical parameters, with clinical outcomes in patients with HCC. Our and serum albumin levels have been previously significantly associated with OS [21, 29, 31], though they were not in the present study.

The radiomics features were derived from CT images both in the late arterial and portal venous phases of the patients. The radiomics signature identified from these features included those both in the late arterial and portal venous phases when building clinical-radiomics and radiomics nomograms for OS, as well as the radiomics nomogram for TTP. However, the radiomics signature only included radiomic features in the late arterial phase for building clinical-radiomics nomogram for TTP. Tumor heterogeneity is closely related to tumor prognosis, most notably in HCC lesions [32]. Heterogeneous arterial phase enhancement seems to be predictive of high tumor grade and recurrence after treatment in HCC. However, advanced HCC often appears to be hypovascular, with decreased arterial flow [33]. This may explain the prognostic significance of radiomics signature, which reflect physiological heterogeneity and can be estimated at the portal venous phase. Our results collectively demonstrate that increased pretreatment tumor 
inhomogeneity is associated with poor clinical outcomes in patients with advanced HCC. These findings are consistent with those of previous HCC studies $[19,21]$.

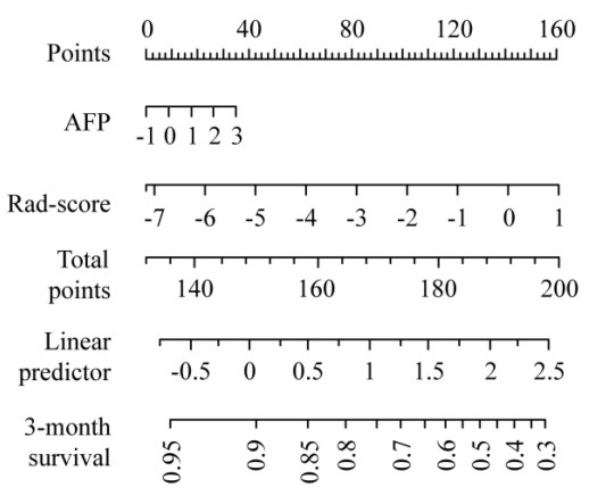

A

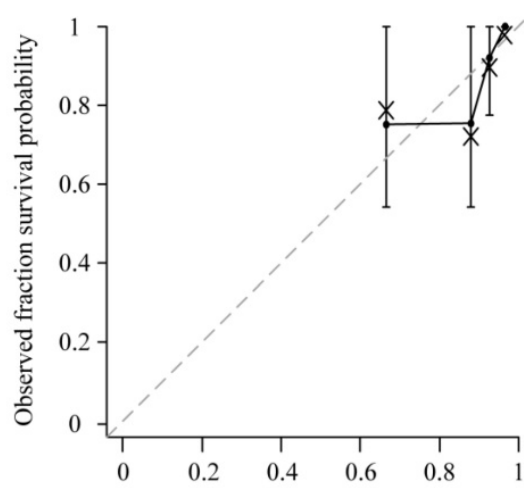

Nomogram predicted survival probability

C

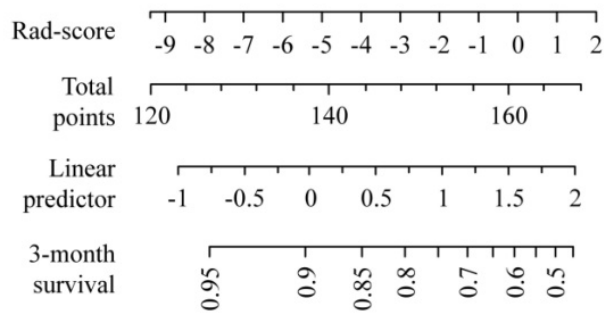

B

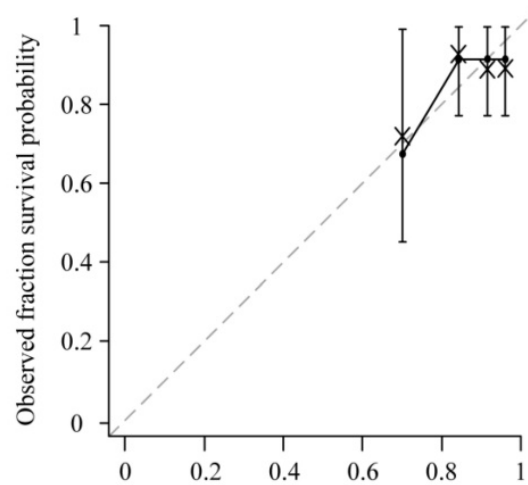

Nomogram predicted survival probability

D

Figure 3. Use of the constructed clinical-radiomics nomogram and radiomics nomogram to predict time to progression (TTP) in patients with advanced HCC, along with the assessment of the model calibration. Clinical-radiomics nomogram (A) and radiomics nomogram (B). Locate the patient's Rad-score on the Rad-score axis. Draw a line straight upward to the points' axis to determine how many points toward the probability of TTP the patient receives for his or her Rad-score. Repeat the process for each variable. Sum the points achieved for each of the risk factors. Locate the final sum on the Total Point axis. Draw a line straight down to find the patient's probability of TTP. Calibration curves for the clinical-radiomics nomogram (C) and radiomics nomogram (D) show the calibration of each model in terms of the agreement between the predicted and the observed 3-month outcomes. Nomogram predicted TTP is plotted on the x-axis; the observed fraction TTP is plotted on the $y$-axis. Diagonal dotted line $=$ a perfect prediction by an ideal model, in which the predicted outcome perfectly corresponds to the actual outcome. Solid line $=$ performance of the nomogram, a closer lining of which with the diagonal dotted line represents a better prediction.

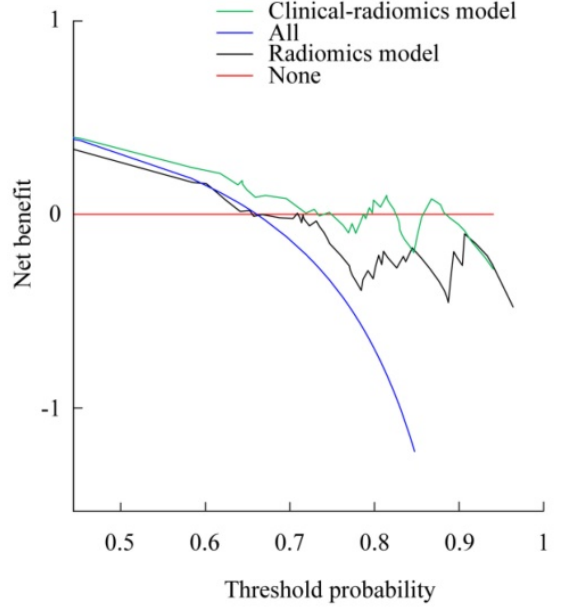

A

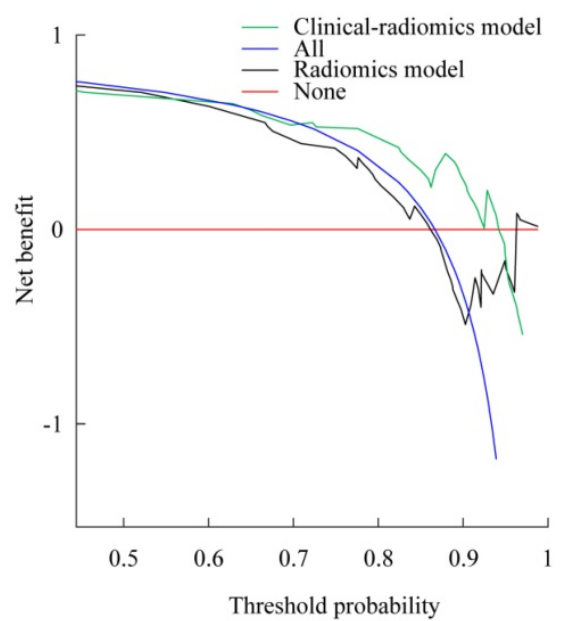

B

Figure 4. Decision curve analysis for each model. Decision curve for the models of prediction overall survival (OS) with l-year survival probability. (B) Decision curve for the models of prediction time to progression (TTP) with 3-month survival probability. The $y$-axis measures the net benefit. The net benefit was calculated by summing the benefits (true-positive results) and subtracting the harms (false-positive results), weighting the latter by a factor related to the relative harm of an undetected cancer compared with the harm of unnecessary treatment. The clinical-radiomics model had the highest net benefit compared with radiomics model and simple strategies such as follow-up of all patients (blue line) or no patients (red line) across the full range of threshold probabilities at which a patient would choose to undergo imaging follow-up. 
As demonstrated in present study, the radiomics signature may predict survival outcomes, supporting the conclusion that radiomics signature can obtain intratumoural heterogeneity in a noninvasive way that is relevant to patient prognosis. Furthermore, the radiomics signature and clinical-radiomics nomogram used here was demonstrated to accurately estimate OS and TTP. When compared with long-term OS outcomes, TTP was defined as a secondary end point associated with less extended follow-up and thus more effective therapeutic adjustment [23]. Thus, the present study proposes an efficient and noninvasive pretreatment prediction tool that enables earlier development of personalized treatment approaches.

The radiomics nomogram, as a statistical model, accounts for multiple risk factors by assigning a total number of points to each patient. A series of studies [26, 34-36] have shown that the radiomics nomogram can effectively and comprehensively predict the post-operative outcomes of individual patients. Furthermore, pretreatment portal venous phasederived tumor entropy may be a predictor of survival in patients with advanced HCC treated with sorafenib [21]. As the first study of a radiomics signature for prediction of OS and TTP in patients with advanced HCC treated with apatinib plus TACE, the present study demonstrated that the use of a clinicalradiomics nomogram achieved superior prognostic performance than a radiomics nomogram alone, with a higher C-index and better calibration. The utility of this proposed nomogram was confirmed in a validation set. A decision curve analysis also revealed that a clinical-radiomics nomogram was superior to a radiomics nomogram across most of the range of reasonable threshold probabilities tested here.

Limitations of present study include its retrospective design, a limited sample size, lack of a control group, and lack of an external model validation. A large-scale independent prospective multicenter validation cohort is thus needed to acquire high-level evidence for broader clinical application. In addition, no gene-expression signature has been incorporated into the nomogram used here. Tumor gene expression patterns can provide insights into patient prognosis [37]. However, intratumor heterogeneity has also led to an underestimation of the tumor genomics landscape, as revealed by single tumor-biopsy samples. This may contribute to treatment failure and drug resistance [38].

\section{Conclusions}

Apatinib plus TACE may improve OS and prolong TTP in the patients with advanced HCC. The clinical-radiomics nomograms, a noninvasive pretreatment prediction tool that incorporate radiomics signature and AFP, demonstrated good prediction accuracy for OS and TTP in these patients. These results indicate that the clinical-radiomics nomograms may provide novel insight for precise personalized medicine approaches in the patients with advanced HCC.

\section{Supplementary Material}

Supplementary figures and tables. http://www.medsci.org/v18p2276s1.pdf

\section{Acknowledgements}

We thank all participants in this study. We greatly appreciate the kind assistance of Huan Liu (GE Healthcare, Shanghai 200000, China) in processing the medical images.

\section{Funding}

This work was supported by grants from the National Natural Science Foundation of China (No. 81873919 and No. 82072041).

\section{Competing Interests}

The authors have declared that no competing interest exists.

\section{References}

1. Akinyemiju T, Abera S, Ahmed M, Alam N, Alemayohu MA, Allen C, et al. The Burden of Primary Liver Cancer and Underlying Etiologies From 1990 to 2015 at the Global, Regional, and National Level: Results From the Global Burden of Disease Study 2015. JAMA oncology. 2017; 3: 1683-91.

2. EASL Clinical Practice Guidelines: Management of hepatocellular carcinoma. Journal of hepatology. 2018; 69: 182-236.

3. Globocan. Fact Sheets by Population-Globocan-IARC. 2017.

4. Park JW, Chen M, Colombo M, Roberts LR, Schwartz M, Chen PJ, et al. Global patterns of hepatocellular carcinoma management from diagnosis to death: the BRIDGE Study. Liver international: official journal of the International Association for the Study of the Liver. 2015; 35: 2155-66.

5. Llovet JM, Ricci S, Mazzaferro V, Hilgard P, Gane E, Blanc J-F, et al. Sorafenib in Advanced Hepatocellular Carcinoma. New England Journal of Medicine. 2008; 359: 378-90.

6. Tian S, Quan H, Xie C, Guo H, Lu F, Xu Y, et al. YN968D1 is a novel and selective inhibitor of vascular endothelial growth factor receptor-2 tyrosine kinase with potent activity in vitro and in vivo. Cancer science. 2011; 102: 1374-80.

7. Xu J, Zhang Y, Jia R, Yue C, Chang L, Liu R, et al. Anti-PD-1 Antibody SHR-1210 Combined with Apatinib for Advanced Hepatocellular Carcinoma, Gastric, or Esophagogastric Junction Cancer: An Open-label, Dose Escalation and Expansion Study. Clinical cancer research: an official journal of the American Association for Cancer Research. 2019; 25: 515-23.

8. Lan CY, Wang Y, Xiong Y, Li JD, Shen JX, Li YF, et al. Apatinib combined with oral etoposide in patients with platinum-resistant or platinum-refractory ovarian cancer (AEROC): a phase 2, single-arm, prospective study. The Lancet Oncology. 2018; 19: 1239-46.

9. Li J, Qin S, Xu J, Xiong J, Wu C, Bai Y, et al. Randomized, Double-Blind, Placebo-Controlled Phase III Trial of Apatinib in patients with Chemotherapy-Refractory Advanced or Metastatic Adenocarcinoma of the Stomach or Gastroesophageal Junction. Journal of clinical oncology: official journal of the American Society of Clinical Oncology. 2016; 34: 1448-54.

10. Liang Q, Kong L, Du Y, Zhu X, Tian J. Antitumorigenic and antiangiogenic efficacy of apatinib in liver cancer evaluated by multimodality molecular imaging. Experimental \& Molecular Medicine. 2019; 51: 76.

11. Qin S. Apatinib in Chinese patients with advanced hepatocellular carcinoma: A phase II randomized, open-label trial. Journal of Clinical Oncology. 2014; 32: 4019.

12. Yang Z, Chen G, Cui Y, Xiao G, Su T, Yu J, et al. The safety and efficacy of TACE combined with apatinib on patients with advanced hepatocellular carcinoma: a retrospective study. Cancer biology \& therapy. 2019; 20: 321-7. 
13. Chen S, Yu W, Zhang K, Liu W. Comparison of the efficacy and safety of Transarterial chemoembolization with and without Apatinib for the treatment of BCLC stage C hepatocellular carcinoma. BMC cancer. 2018; 18: 1131.

14. Lu W, Jin XL, Yang C, Du P, Jiang FQ, Ma JP, et al. Comparison of efficacy between TACE combined with apatinib and TACE alone in the treatment of intermediate and advanced hepatocellular carcinoma: A single-center randomized controlled trial. Cancer biology \& therapy. 2017; 18: 433-8.

15. Zhu K, Chen J, Lai L, Meng X, Zhou B, Huang W, et al. Hepatocellular carcinoma with portal vein tumor thrombus: treatment with transarterial chemoembolization combined with sorafenib--a retrospective controlled study. Radiology. 2014; 272: 284-93.

16. Choi GH, Shim JH, Kim MJ, Ryu MH, Ryoo BY, Kang YK, et al. Sorafenib alone versus sorafenib combined with transarterial chemoembolization for advanced-stage hepatocellular carcinoma: results of propensity score analyses. Radiology. 2013; 269: 603-11.

17. Kan X, Liang B, Zhou G, Xiong B, Pan F, Ren Y, et al. Transarterial Chemoembolization Combined With Apatinib for Advanced Hepatocellular Carcinoma: A Propensity Score Matching Analysis. Front Oncol. 2020; 10: 970.

18. Gillies RJ, Kinahan PE, Hricak H. Radiomics: Images Are More than Pictures, They Are Data. Radiology. 2016; 278: 563-77.

19. Xu X, Zhang HL, Liu QP, Sun SW, Zhang J, Zhu FP, et al. Radiomic Analysis of Contrast-Enhanced CT Predicts Microvascular Invasion and Outcome in Hepatocellular Carcinoma. Journal of hepatology. 2019; 70: 1133-44.

20. Kim S, Shin J, Kim DY, Choi GH, Kim MJ, Choi JY, Radiomics on gadoxetic acid-enhanced magnetic resonance imaging for prediction of postoperative early and late recurrence of single hepatocellular carcinoma. Clinical cancer research : an official journal of the American Association for Cancer Research. 2019; 25: 3847-55.

21. Mule S, Thiefin G, Costentin C, Durot C, Rahmouni A, Luciani A, et al. Advanced Hepatocellular Carcinoma: Pretreatment Contrast-enhanced CT Texture Parameters as Predictive Biomarkers of Survival in Patients Treated with Sorafenib. Radiology. 2018; 288: 445-55.

22. Llovet JM, Di Bisceglie AM, Bruix J, Kramer BS, Lencioni R, Zhu AX, et al. Design and endpoints of clinical trials in hepatocellular carcinoma. Journal of the National Cancer Institute. 2008; 100: 698-711.

23. Lencioni R, Llovet JM. Modified RECIST (mRECIST) assessment for hepatocellular carcinoma. Seminars in liver disease. 2010; 30: 52-60.

24. Shrout PE, Fleiss JL. Intraclass correlations: uses in assessing rater reliability. Psychological bulletin. 1979; 86: 420-8.

25. Li L, Fan W, Li J, Li Q, Wang J, Fan Y, et al. Abnormal brain structure as a potential biomarker for venous erectile dysfunction: evidence from multimodal MRI and machine learning. Eur Radiol. 2018; 28: 3789-800.

26. Huang YQ, Liang CH, He L, Tian J, Liang CS, Chen X, et al. Development and Validation of a Radiomics Nomogram for Preoperative Prediction of Lymph Node Metastasis in Colorectal Cancer. Journal of clinical oncology : official journal of the American Society of Clinical Oncology. 2016; 34: 2157-64.

27. Kerr KF, Brown MD, Zhu K, Janes H. Assessing the Clinical Impact of Risk Prediction Models With Decision Curves: Guidance for Correct Interpretation and Appropriate Use. Journal of clinical oncology: official journal of the American Society of Clinical Oncology. 2016; 34: 2534-40.

28. Cheng A-L, Kang Y-K, Chen Z, Tsao C-J, Qin S, Kim JS, et al. Efficacy and safety of sorafenib in patients in the Asia-Pacific region with advanced hepatocellular carcinoma: a phase III randomised, double-blind, placebocontrolled trial. The Lancet Oncology. 2009; 10: 25-34.

29. Tandon P, Garcia-Tsao G. Prognostic indicators in hepatocellular carcinoma: a systematic review of 72 studies. Liver international: official journal of the International Association for the Study of the Liver. 2009; 29: 502-10.

30. Shanbhogue AK, Prasad SR, Takahashi N, Vikram R, Sahani DV. Recent Advances in Cytogenetics and Molecular Biology of Adult Hepatocellular Tumors: Implications for Imaging and Management. Radiology. 2011; 258: 673-93.

31. Llovet JM, Ricci S, Mazzaferro V, Hilgard P, Gane E, Blanc JF, et al. Sorafenib in advanced hepatocellular carcinoma. The New England journal of medicine. 2008; 359: 378-90.

32. Segal E, Sirlin CB, Ooi C, Adler AS, Gollub J, Chen X, et al. Decoding global gene expression programs in liver cancer by noninvasive imaging. Nature biotechnology. 2007; 25: 675-80.

33. Choi JY, Lee JM, Sirlin CB. CT and MR imaging diagnosis and staging of hepatocellular carcinoma: part I. Development, growth, and spread: key pathologic and imaging aspects. Radiology. 2014; 272: 635-54.

34. Huang Y, Liu Z, He L, Chen X, Pan D, Ma Z, et al. Radiomics Signature: A Potential Biomarker for the Prediction of Disease-Free Survival in Early-Stage (I or II) Non-Small Cell Lung Cancer. Radiology. 2016; 281: 947-57.

35. Wu S, Zheng J, Li Y, Yu H, Shi S, Xie W, et al. A Radiomics Nomogram for the Preoperative Prediction of Lymph Node Metastasis in Bladder Cancer. Clinical cancer research: an official journal of the American Association for Cancer Research. 2017; 23: 6904-11.

36. Han L, Zhu Y, Liu Z, Yu T, He C, Jiang W, et al. Radiomic nomogram for prediction of axillary lymph node metastasis in breast cancer. Eur Radiol. 2019; 29: 3820-9.

37. Segal E, Friedman N, Kaminski N, Regev A, Koller D. From signatures to models: understanding cancer using microarrays. Nature genetics. 2005; 37 Suppl: S38-45.
38. Gerlinger M, Rowan AJ, Horswell S, Math M, Larkin J, Endesfelder D, et al. Intratumor heterogeneity and branched evolution revealed by multiregion sequencing. The New England journal of medicine. 2012; 366: 883-92. 